\title{
Diseño de una metodología para generar un plan de mantenimiento a través de la integración de RCM, WCM y Lean Manufacturing aplicable en procesos de trefilado de alambrón ${ }^{1}$
}

\section{Design of a methodology to generate a maintenance plan through the integration of RCM, WCM and Lean Manufacturing in wire drawing processes}

\author{
E. Pérez, I. A. Castiblanco y N. F. Mateo \\ Recibido: Julio 26 de 2019 - Aceptado: Junio 25 de 2020
}

\begin{abstract}
Resumen - Se presenta el desarrollo de cinco fases basadas en el Design Thinking (Empatizar, Definir, Idear, Prototipar, Evaluar) con el propósito de diseñar una metodología integrando herramientas de Reliability Centered Maintenance (RCM), World Class Manufacturing (WCM) y Lean Manufacturing, la cual permita generar un plan de mantenimiento aplicable en procesos de trefilado de alambrón particularmente en una empresa mexicana del sector del acero. En las fases "Empatizar, Definir e Idear", se identifican los problemas principales en procesos del sector del acero de empresas colombianas y una en México, planteando alternativas de solución basadas en las necesidades y realidades, considerando los Key Perfomance Indicators (KPI) esperados. Posteriormente se seleccionan herramientas acordes al problema y a las alternativas de solución planteadas. En las fases "Prototipar y Evaluar", se integran las herramientas seleccionadas generando un plan de mantenimiento el cual es descrito y representado por un diagrama como evaluación de la metodología diseñada.
\end{abstract}

${ }^{1}$ Producto derivado del proyecto de investigación Diseño de una metodología para generar un plan de mantenimiento a través de la integración de reliability centered maintenance, world class manufacturing y lean manufacturing aplicable en procesos de trefilado de alambrón", apoyado por la Escuela Colombiana De Ingeniería Julio Garavito a través de la Maestría en Ingeniería industrial.

E. Pérez, Escuela Colombiana de Ingeniería Julio Garavito, Bogotá D.C., Colombia, emilio.adan@hotmail.com.

I. A. Castiblanco, Politécnico di Torino, Turín, Italia, ivonne.castiblanco@polito.it

N. F. Mateo, Universidad del Caribe, Cancún, Q. Roo, México, nmateo@ucaribe.edu.mx

Como citar este artículo: Pérez, E., Castiblanco, I. A., y Mateo, N. F. Diseño de una metodología para generar un plan de mantenimiento a través de la integración de RCM, WCM y Lean Manufacturing aplicable en procesos de trefilado de alambrón, Entre Ciencia e Ingeniería, vol. 14, no. 27, pp. 82-90, enero-junio 2020. DOI: https://doi.org/10.31908/19098367.1793.

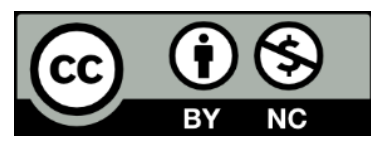

Attribution-NonCommercial 4.0 Intenational (CC By-NC 4.0)
Palabras clave - Mantenimiento centrado en confiabilidad, manufactura de clase mundial, Manufactura esbelta, plan de mantenimiento.

Abstract - The development of five phases based on Design Thinking (Empathize, Define, Ideate, Prototype, Evaluate) is presented in order to design a methodology integrating Reliability Centered Maintenance (RCM), World Class Manufacturing (WCM) and Lean Manufacturing tools, which allows generating a maintenance plan applicable in wire drawing processes, particularly in a Mexican company in the steel sector. In the "Empathize, Define and Devise" phases, the main problems in processes of the steel sector of Colombian companies and one in Mexico are identified, proposing solution alternatives based on needs and realities, considering the expected Key Performance Indicators (KPI). Subsequently, tools are selected according to the problem and the alternative solutions proposed. In the "Prototype and Evaluate" phases, the selected tools are integrated, generating a maintenance plan which is described and represented by a diagram as an evaluation of the designed methodology.

Keywords-Lean manufacturing, maintenance plan, reliability centered maintenance, world class manufacturing.

\section{INTRODUCCIÓN}

$\mathrm{H}$ OY en día los fabricantes en las industrias están comprometidos a proporcionar productos de calidad con alto rendimiento para permanecer en el mercado y enfrentar la fuerte presión competitiva en todo el mundo, produciendo a un costo mínimo. Ante esta situación surge la necesidad de buscar nuevas alternativas para mejorar las operaciones de mantenimiento en los procesos de trefilados de alambrón en el sector del acero.

El estudio se centra en el análisis de empresas del sector del acero en Colombia y una empresa en México dedicada al trefilado de alambrón; ésta última, en el periodo del $22 / 11 / 2016$ al 31/01/18 presentó paros por averías en sus equipos con un índice de $87.83 \%$ del total de maquinaria, lo que se ha reflejado en la rentabilidad de la misma. En esta 
organización el mantenimiento se ha desarrollado por personal que cuenta con experiencia en el área, esto ha ocasionado paradigmas al llevar a cabo adecuadamente las tareas de mantenimiento.

Por tal motivo, en la presente investigación se analizan diversas herramientas de Reliability Centered Maintenance (RCM), World Class Manufacturing (WCM) y Lean Manufacturing, con el propósito de diseñar una metodología a través de la integración de dichas herramientas, la cual permita generar un plan de mantenimiento para los equipos de producción del proceso de trefilado de alambrón particularmente de una empresa del sector del acero en México.

Se muestra el desarrollo de cinco etapas que propone el Design Thinking las cuales se consideran necesarias para el logro de los objetivos planteados. La selección de las herramientas se lleva a cabo considerando que estas metodologías muestran un enfoque directo en el tema de estudio (mantenimiento) y desarrollo del factor humano como elemento fundamental.

Con esta propuesta se contribuye a mejorar el plan de mantenimiento en los equipos de producción de una empresa mexicana del sector del acero, considerando que la aplicación de dicha metodología con estudios posteriores pueda llevarse a cabo en empresas de Colombia y otras empresas del mismo sector.

\section{METODOLOGÍA}

Para llevar a cabo la integración de las herramientas se tomó apoyo de una metodología de diseño, para ello fue necesario analizar diversas metodologías de diseño tales como: Ulrich, Schnarch, APQP, Design Thinking (Stanford) y Hoshin Kanri, las cuales muestran procedimientos enfocados al diseño y desarrollo de productos. Sin embargo, el desarrollo de esta investigación estará basado en el Design Thinking, dado que este método muestra un enfoque particular en el factor humano de las organizaciones, considerando adicionalmente, que en la actualidad es uno de los métodos más utilizados por diseñadores para pensar y solucionar problemas en las empresas en Europa, Asia y Estados Unidos [1].

Fase 1 Empatizar: Aplicación de encuestas a empresas del sector del acero en Colombia y una empresa mexicana.

En esta fase se aplicó una encuesta cualitativa a una empresa mexicana del sector del acero, así como a 18 empresas colombianas del mismo sector para identificar los principales problemas que han enfrentado en sus procesos de trefilado de alambrón, el tipo de mantenimiento que desarrollan y el conocimiento que tienen sobre las metodologías RCM, WCM y Lean Manufacturing, así como las dificultades que pueda presentar el personal sobre la adaptación a una nueva forma de trabajo, los resultados se observan en las fig. 1,2,3 y 4.

1.- ¿Cuáles han sido los problemas que ha experimentado usted en su área de producción?

El 79\% de las empresas encuestadas presentan problemas en el mantenimiento de los equipos de producción, sin embargo, el $16 \%$ mencionó que enfrentan problemas de calidad, lo que es posible considerar que el funcionamiento de los equipos de producción contribuye a la calidad de los productos.

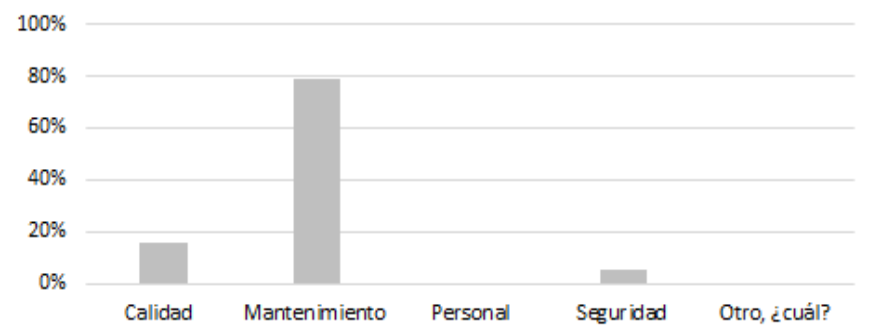

Fig. 1. Problemas relevantes que enfrentan las empresas encuestadas [2].

La fig. 2 muestra el porcentaje de las empresas encuestadas que tienen conocimiento sobre herramientas de mejora para sus procesos, por lo que es un factor importante que se debe considerar al desarrollar la metodología.

2.- ¿Conoce usted alguna herramienta para la mejora continua?

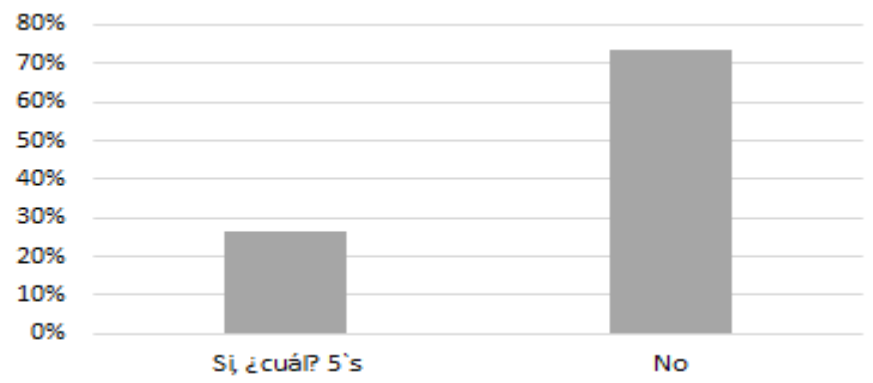

Fig. 2. Conocimiento de las empresas sobre herramientas de mejora [2].

El $26 \%$ de las empresas encuestadas mencionaron que conocen y han implementado la metodología de las $5 \mathrm{~s}$ y que ésta ha favorecido en algunos cambios en las áreas de trabajo, mientras que el $74 \%$ no tienen conocimiento alguno sobre metodologías mencionadas.

3.- ¿Qué tipo de mantenimiento desarrollan en sus equipos de producción?

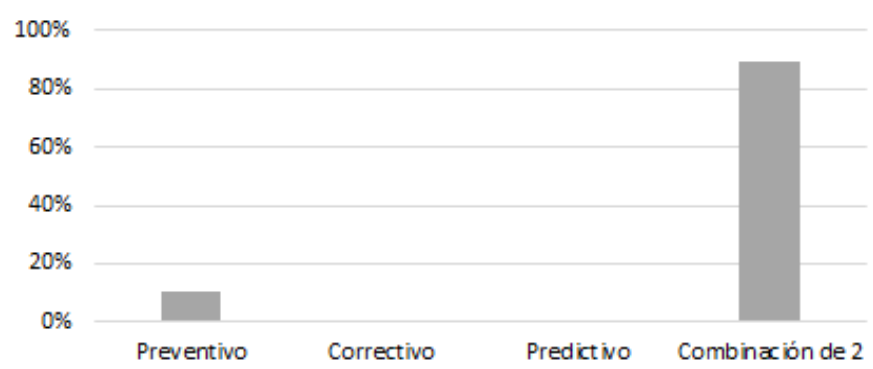

Fig. 3. Tipos de mantenimiento [2].

En la fig. 3 se observa que el $89 \%$ de las empresas que se encuestaron realizan la combinación del mantenimiento preventivo y correctivo en sus equipos de producción, el $11 \%$ trabajan en la aplicación del mantenimiento preventivo, sin embargo, mencionan que han enfrentado acciones correctivas. La resistencia al cambio es un aspecto importante para la implementación de herramientas de mejora, este factor se evalúa a través de la siguiente interrogante donde el $63 \%$ mencionó que no habría resistencia a cambiar la forma de trabajo de mantenimiento, mientras que el resto comentó que habría resistencia, pero apoyan el cambio, los resultados se 
observan en la fig. 4.

4.- ¿Cómo considera usted la resistencia al cambio de los empleados con respecto a la implementación de un nuevo plan de mantenimiento?

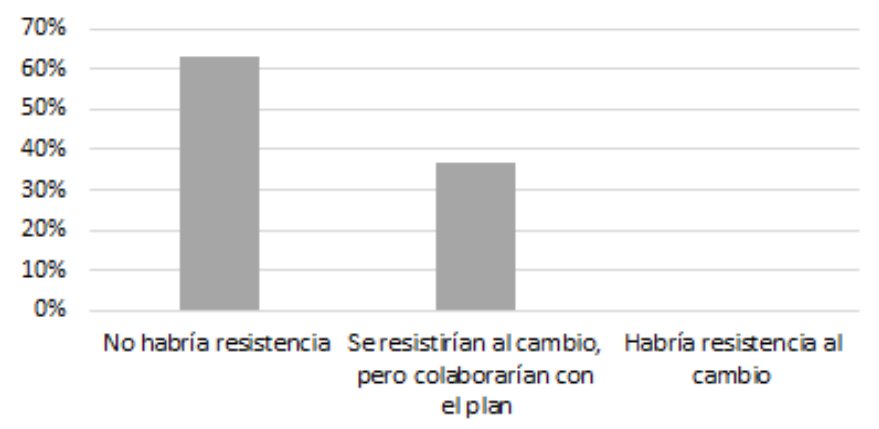

Fig. 4. Evaluación sobre la resistencia a la implementación del plan [2].

Para el caso de la empresa mexicana del sector del acero, se realiza un análisis partiendo de una base de datos sobre el número fallas en los equipos de producción durante el periodo del 22/11/2016 al 31/01/18. El área piloto que se asignó para el desarrollo del plan de mantenimiento es el área de trefilado de alambrón, la tabla I muestra el registro de las máquinas y el número de fallos en el periodo mencionado

TABLA I.

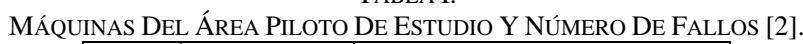

\begin{tabular}{|r|c|}
\hline MÁQUINAS & FRECUENCIA DE FALLO \\
\hline BULL 2 & 157 \\
\hline BULL 1 & 129 \\
\hline BULL 4 & 57 \\
\hline BULL 7 & 25 \\
\hline BULL 3 & 20 \\
\hline BULL 6 & 12 \\
\hline
\end{tabular}

\section{REVISIÓN DE LA LITERATURA}

Un aspecto importante en el desarrollo de la investigación es tener el contexto literario sobre las herramientas de las metodologías RCM, WCM, y Lean Manufacturing, por tal motivo, a continuación, se describen dichas metodologías.

\section{A. Herramientas de mejora continua de Lean} Manufacturing.

Lean Manufacturing consiste en la aplicación sistemática y habitual de diferentes herramientas para el mejoramiento de los procesos productivos [3]. La tabla II muestra un conjunto de herramientas de Lean Manufacturing, así como la aplicación / objetivo que sigue cada una.

\section{B. Pilares técnicos de WCM}

Yamashina, Stefan [4] \& De Felice, Petrillo [5] definen 10 pilares técnicos en WCM, además, mencionan que los niveles de logro en los campos técnicos se ven afectados indirectamente por el nivel de logro en los campos administrativos. En la tabla III se muestran los pilares técnicos de WCM y sus objetivos en la implementación, entre estos pilares se encuentra el AM y el PM los cuales serán considerados para el diseño de la metodología debido a que estos están estrechamente relacionados con el tema de estudio.

\section{Herramientas de RCM}

En el mismo sentido el RCM es un enfoque de mejora industrial centrado en la identificación y el establecimiento de las operaciones de mantenimiento, así como en las políticas de mejoras que gestionan los riesgos de fallas en los equipos. Se observa que la máquina BULL 2 presentó la mayor cantidad de fallos en el periodo mencionado (la máquina BULL 5 no presentó fallos correctivos en dicho periodo). Considerando los resultados obtenidos de la aplicación de las encuestas a una empresa mexicana del sector del acero y a 18 empresas en Colombia del mismo sector, se pudo observar que el $79 \%$ de esta muestra encuestada enfrentan problemas en el mantenimiento de sus equipos, permitiendo un desarrollo de mantenimiento completo. De la misma forma que WCM y Lean Manufacturing requieren de un conjunto de herramientas para su implementación; para el caso del RCM algunos autores han aplicado dicha metodología usando herramientas y estrategias acordes al caso de estudio [6].

En la tabla IV se observa una relación de autores y herramientas que estos han implementado para dar solución a las siete preguntas básicas que intenta resolver el RCM durante el desarrollo de su aplicación [7].

\section{Fase 2 Definir: Definición del problema con enfoque directo considerando KPI.}

Como se mencionó en la fase 1, el problema principal identificado en empresas de Colombia a través de la aplicación de encuestas y revisión literaria se encuentra en el área de mantenimiento de los equipos en los diferentes procesos de producción. Sin embargo, los resultados también muestran que las empresas poseen poco conocimiento sobre la aplicación de herramientas de mejora continua tal como lo muestra la fig. 2 y en consecuencia el desempeño de los KPI se ha visto afectado. Para el caso de la empresa mexicana el problema principal radica en el área de trefilado de alambrón y se observa en la tabla I que la máquina BULL 2 presentó mayor índice de mantenimientos correctivos (fallas) en el periodo mencionado.

Fase 3 Idear: Selección de herramientas de RCM, WCM y Lean Manufacturing para el diseño de la metodología.

Por otra parte, considerando que las metodologías RCM, WCM y Lean Manufacturing contienen herramientas que combinadas pueden ofrecer resultados satisfactorios [4] para los procesos que desarrollan las empresas del sector del acero. La selección de las herramientas en esta fase se realiza considerando aquellas que tienen relación directa con elementos tales como: el objetivo de la investigación, el problema identificado en la fase 1 y los enfoques 1 y 2 que se describen a continuación y la revisión de la literatura.

La fig. 5 muestra la relación directa (D) e indirecta (I) que tiene cada herramienta de Lean Manufaturing con los elementos mencionados, de la misma forma se observa en la fig. 6 la relación "D" e "I" de las herramientas de WCM con dichos elementos, y posteriormente la fig. 7 presenta la relación de las herramientas aplicadas en la implementación del RCM con los elementos antes mencionados. 
TABLA II.

HERRAMIENTAS DE LEAN MANUFACTURING [2].

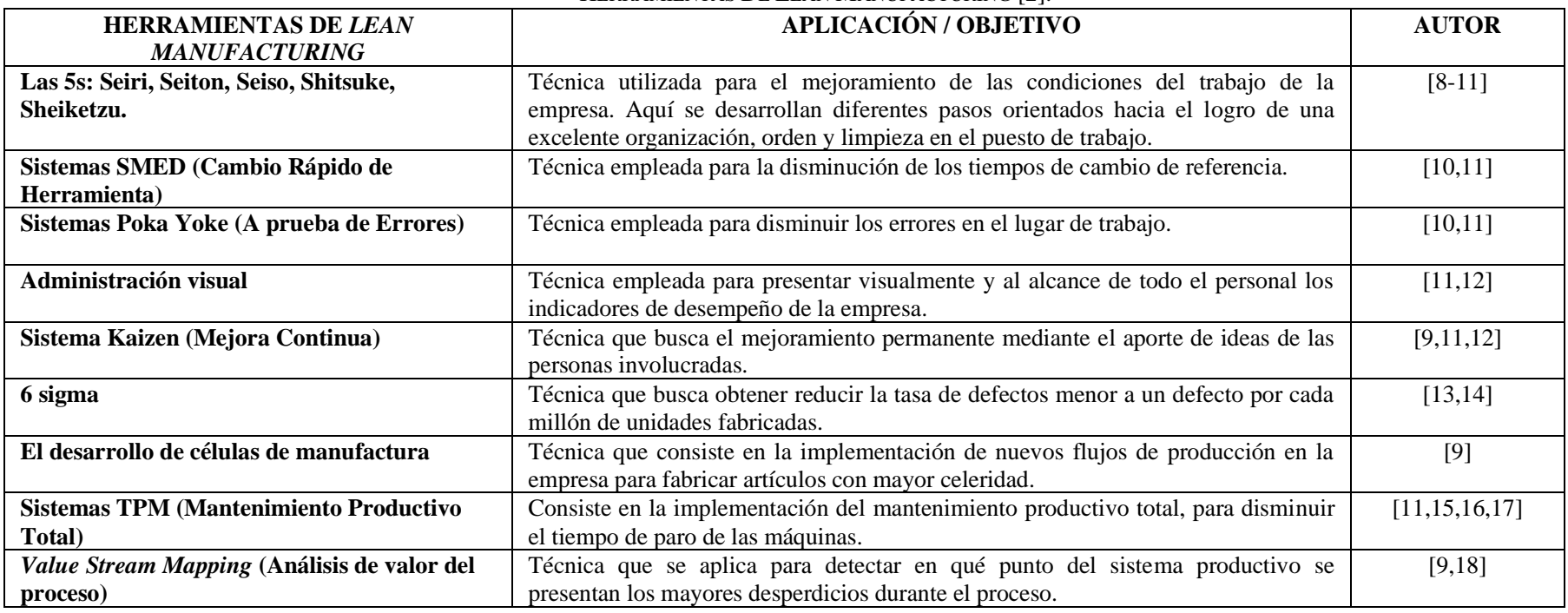

TABLA III.

Pilares Técnicos De WCM [2].

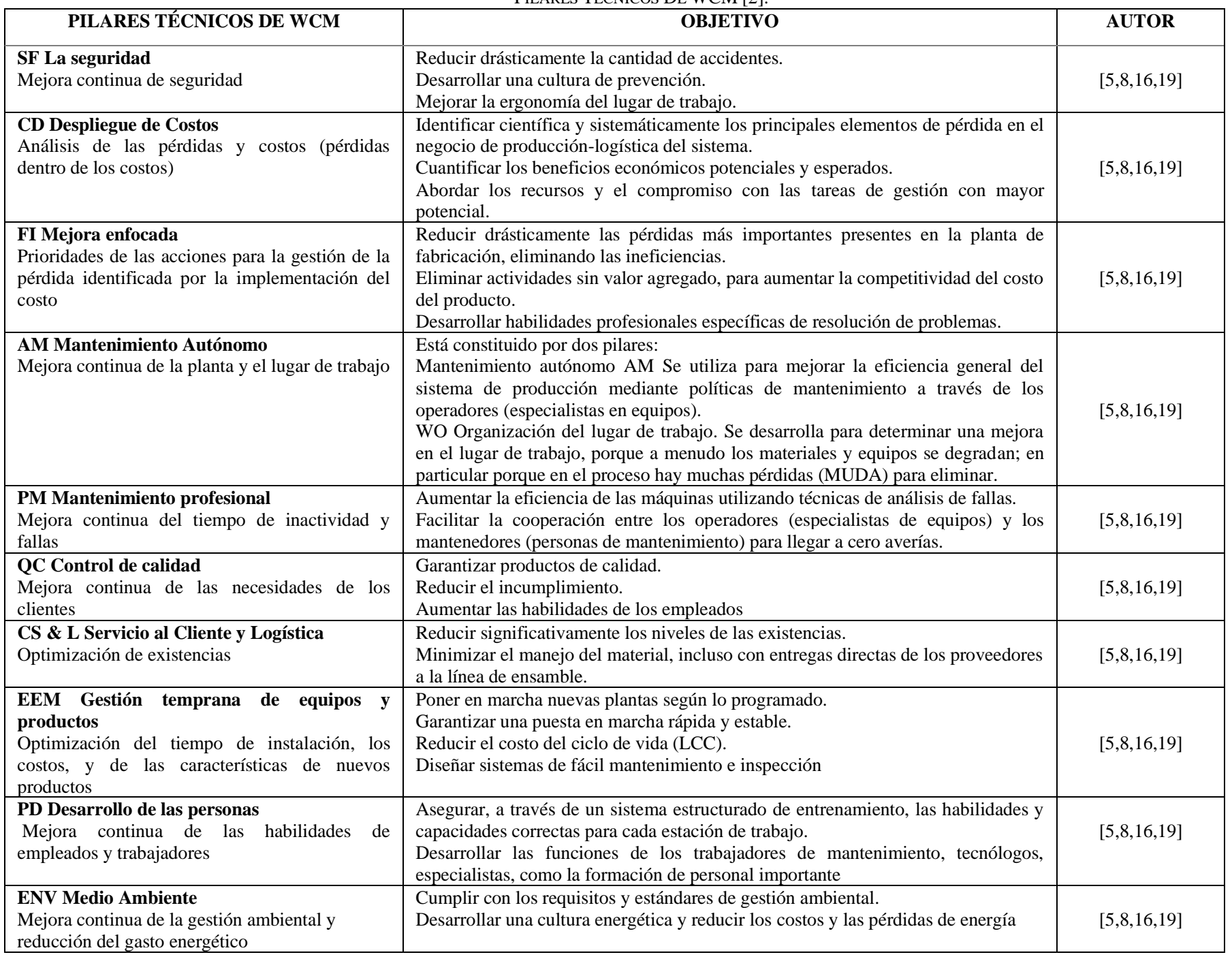


TABLA IV

HERRAMIENTAS UTILIZADAS EN CASO DE AMPLIACIÓN DE RCM [2].

\begin{tabular}{|c|c|c|c|c|c|}
\hline \multirow{3}{*}{ PREGUNTAS RCM } & \multicolumn{5}{|c|}{ AUTORES / CASOS DE APLICACIÓN } \\
\hline & [20] & & {$[21]$} & {$[22]$} & [23] \\
\hline & \multicolumn{5}{|c|}{ HERRAMIENTAS Y ESTRATEGIAS UTILIZADAS } \\
\hline ¿Cuáles son las funciones? & DFP, PMA, & $\mathrm{HC}$ & DFP & AMEF & AF, PMA \\
\hline ¿De qué manera puede fallar? & $\mathrm{HC}$ & & AMEF & AMEF & AMEF \\
\hline ¿Cuál es la causa de la falla? & & & RHF & AMEF & AMEF \\
\hline ¿Qué sucede cuando ocurre cada falla? & & & RHF & AMEF & \\
\hline ¿En qué sentido cada falla es importante? & $\mathrm{AD}, \mathrm{HC}$ & & MANC, AAF & & $\mathrm{ACR}, \mathrm{AAF}$ \\
\hline ¿Qué se puede hacer para prevenir cada falla? & $\mathrm{AD}, \mathrm{HC}$ & & RM & MBC, FD-RN & F-DW, D-SM \\
\hline $\begin{array}{l}\text { ¿Qué debe hacerse si no se puede encontrar una tarea } \\
\text { preventiva adecuada? }\end{array}$ & & & PRP & & \\
\hline \multicolumn{6}{|c|}{ DESCRIPCIÓN DE HERRAMIENTAS } \\
\hline \multicolumn{2}{|c|}{$\begin{array}{l}\text { PMA: Programas de mantenimiento actuales } \\
\text { HC: Hoja de cálculo } \\
\text { AD: Árbol de decisión } \\
\text { DFP: Diagrama de flujo del proceso } \\
\text { AMEF: Análisis de modos y efectos de fallas } \\
\text { RHF: Registros históricos de fallas } \\
\text { MANC: Matriz de asignación de nivel crítico } \\
\text { PRP: Programa retroalimentación personal }\end{array}$} & \multicolumn{4}{|c|}{$\begin{array}{ll}\text { MBC: } & \text { Mantenimiento basado en la condición } \\
\text { FD-RN: Fusión de datos redes neuronales } \\
\text { AF: } & \text { Árbol funcional } \\
\text { ACR: } & \text { Análisis de causa raíz } \\
\text { F-DW: } & \text { Fiabilidad con distribución de Weibull } \\
\text { D-SM: } & \text { Disponibilidad con simulación Monte Carlo. } \\
\text { AAF: } & \text { Árbol de análisis de falla. } \\
\text { RM: } & \text { Mantenimiento radical }\end{array}$} \\
\hline
\end{tabular}

PROBLEMAS IDENTIFICADOS

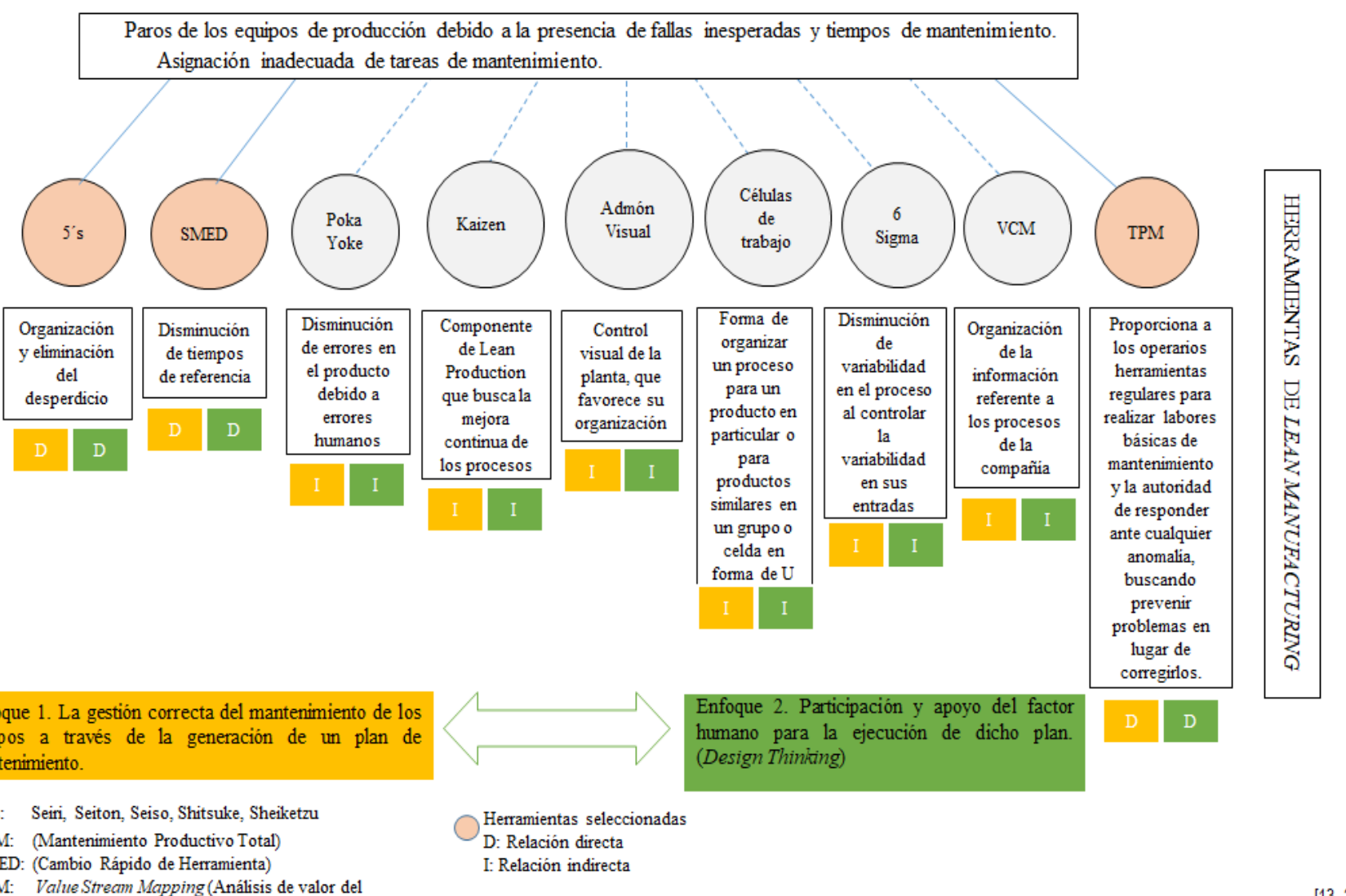

TPM: (Mantenimiento Productivo Total)

VSM: Value Stream Mapping (Análisis de valor del proceso)

Fig. 5. Relación de herramientas de Lean Mamufacturin \& problema de estudio (continuación) 
PROBLEMAS IDENTIFICADOS

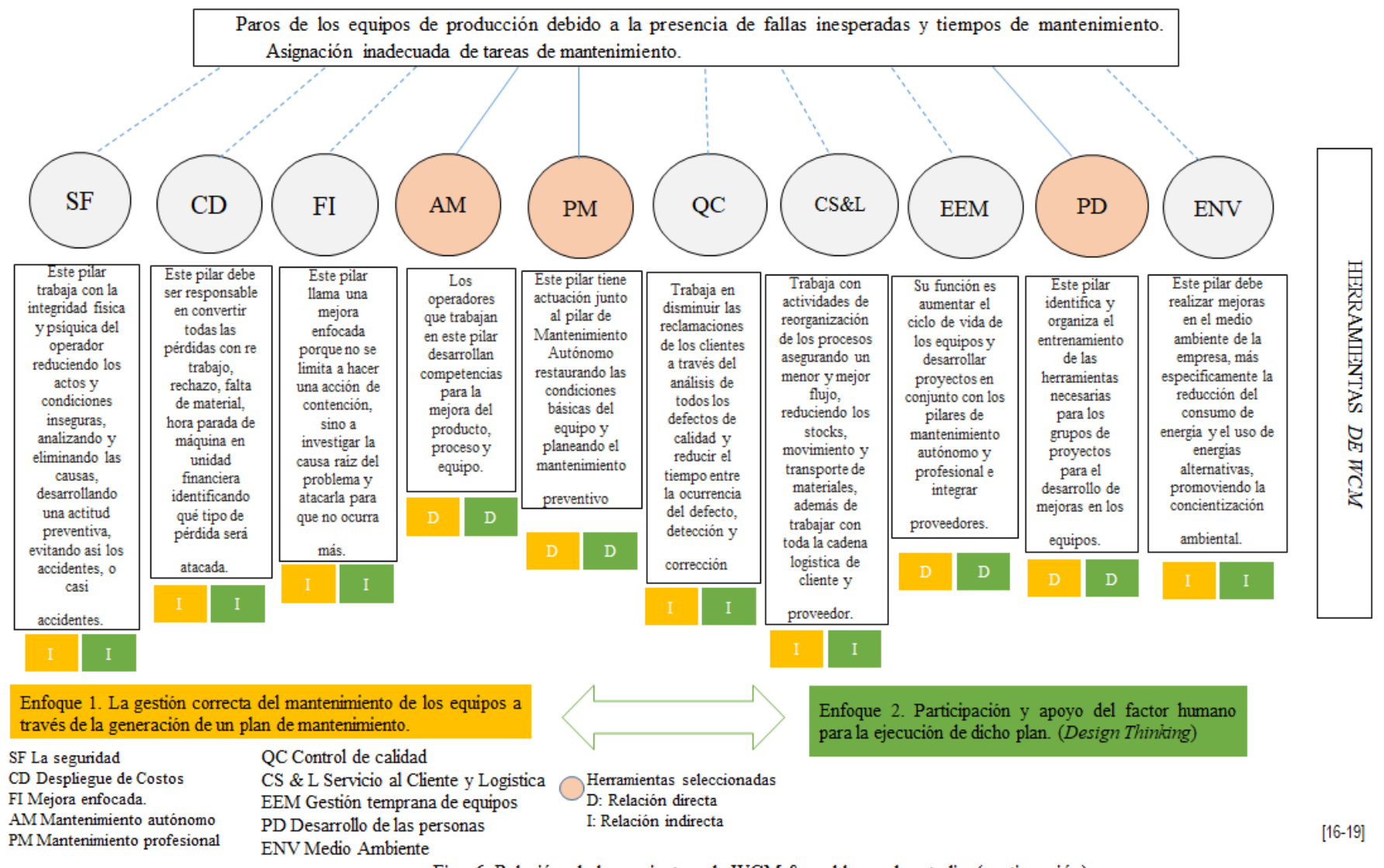

Fig. 6. Relación de herramientas de WCM \& problema de estudio (continuación)

PROBLEMAS IDENTIFICADOS
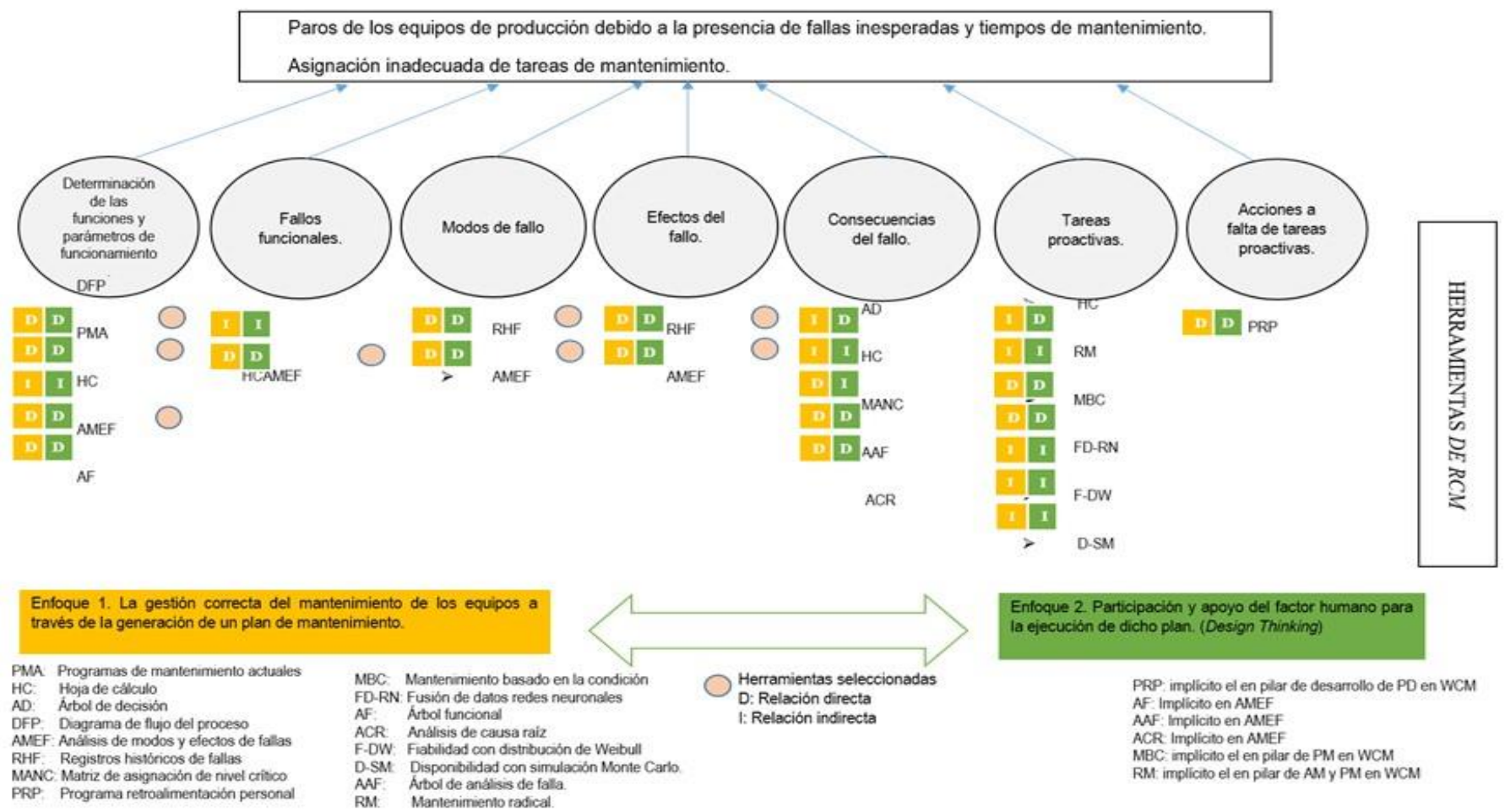

la ejecución de dicho plan. (Design Thinking)

PRP implicito el en pilar de desarrollo de PD en WCM AF: Implicito en AMEF

AAF: Implicito en AMEF

ACR Implicito en AMEF

MBC: implicio el en pilar de PM en WCM

RM. implicito el en plar de AM y PM en WCM

Fig. 1. Relación de herramientas de RCM \& problema de estudio (continuación). 
La selección de herramientas en esta investigación se llevó a cabo considerando dos enfoques principales:

Enfoque 1. La gestión correcta del mantenimiento de los equipos a través de la generación de un plan de mantenimiento. (Herramientas de RCM, WCM y Lean Manufacturing).

Enfoque 2. Participación y apoyo del factor humano para la ejecución de dicho plan. (Design Thinking)

Es preciso mencionar que algunas herramientas de RCM no fueron seleccionadas ya que se considera que están implícitas en alguno de los pilares de WCM o Lean Manufacturing, la clasificación de las herramientas seleccionadas se muestra en la tabla V.

TABLA V

HERRAMIENTAS SELECCIONADAS

\begin{tabular}{|l|c|c|}
\hline \multicolumn{3}{|c|}{ HERRAMIENTAS SELECCIONADAS } \\
\hline $\begin{array}{l}\text { Lean } \\
\text { Manufacturing }\end{array}$ & WCM & RCM \\
\hline$>$ Las 5s & $>$ AM Mantenimiento & $>$ PMA: Programas \\
$>$ Sistemas SMED & autónomo & de mantenimiento \\
$>$ Sistemas TPM & $>$ PM Mantenimiento & actuales \\
& profesional & $>$ DFP: Diagrama de \\
& $>$ PD Desarrollo de & flujo del proceso \\
& las personas & $>$ AMEF: Análisis de \\
& modos y efectos \\
& & de fallas \\
& & RHF: Registros \\
& & históricos de fallas \\
\hline
\end{tabular}

Fase 4. Diseño de la metodología a través de la Integración de herramientas de RCM, WCM y Lean Manufacturing. Etapa de resultados: Fase 4 y 5. De acuerdo con [Gonzalez [24]] en la fase 4 del proceso de Design Thinking, Prototipar "es la generación de elementos informativos como dibujos, objetos o artefactos, con los que se pretende responder las inquietudes que nos acercan a la solución final'. Una vez identificado el problema y/o las oportunidades de mejora en los procesos de producción a partir de la aplicación de encuestas cualitativas a diferentes empresas del sector del acero, se integran las herramientas que se consideraron necesarias para continuar

con el diseño de la metodología, generando prototipos basados en las aplicaciones de las herramientas WCM, RMC y Lean Manufacturing, tales como: diagramas o esquemas para el desarrollo adecuado de tareas de mantenimiento tomando como caso de estudio el proceso de trefilado de alambrón de la empresa mexicana ya mencionada, así como el problema identificado en las encuestas realizadas y considerando además, el objetivo principal de la presente investigación.

La fig. 8 muestra un diagrama general de los objetivos de la investigación; las tres metodologías de la izquierda se muestran unidas para generar el diseño de una metodología a través de la cual se generará un plan de mantenimiento, lo anterior integrado con base en el Design Thinking.

En esta fase se muestran los resultados esperados de la investigación tales como: diseño de una metodología para generar un plan de mantenimiento la cual se muestra en la fig. 9, plan de mantenimiento y prototipos para la realización de tareas de mantenimiento (diagramas).
Fase 5. Plan de mantenimiento para el proceso de trefilado de alambrón de una empresa mexicana.

Esta etapa consiste en generar y evaluar prototipos, considerando como prototipos en esta investigación a los diagramas o esquemas que permiten el flujo adecuado de actividades del mantenimiento, además de ser otra oportunidad para ganar empatía por las personas y refinar las soluciones y poder mejorarlas [28]. En la fig. 9 se muestra la metodología propuesta para generar un plan de mantenimiento piloto aplicable en una empresa mexicana del sector del acero. A partir de la metodología se desarrolla un modelo que muestra el seguimiento de las actividades de mantenimiento particularmente del proceso de trefilado de alambrón de una empresa mexicana. En la fig. 10 se observan las tres etapas de la metodología de mantenimiento, así como la serie de pasos necesarios para llevar a cabo el plan de mantenimiento.

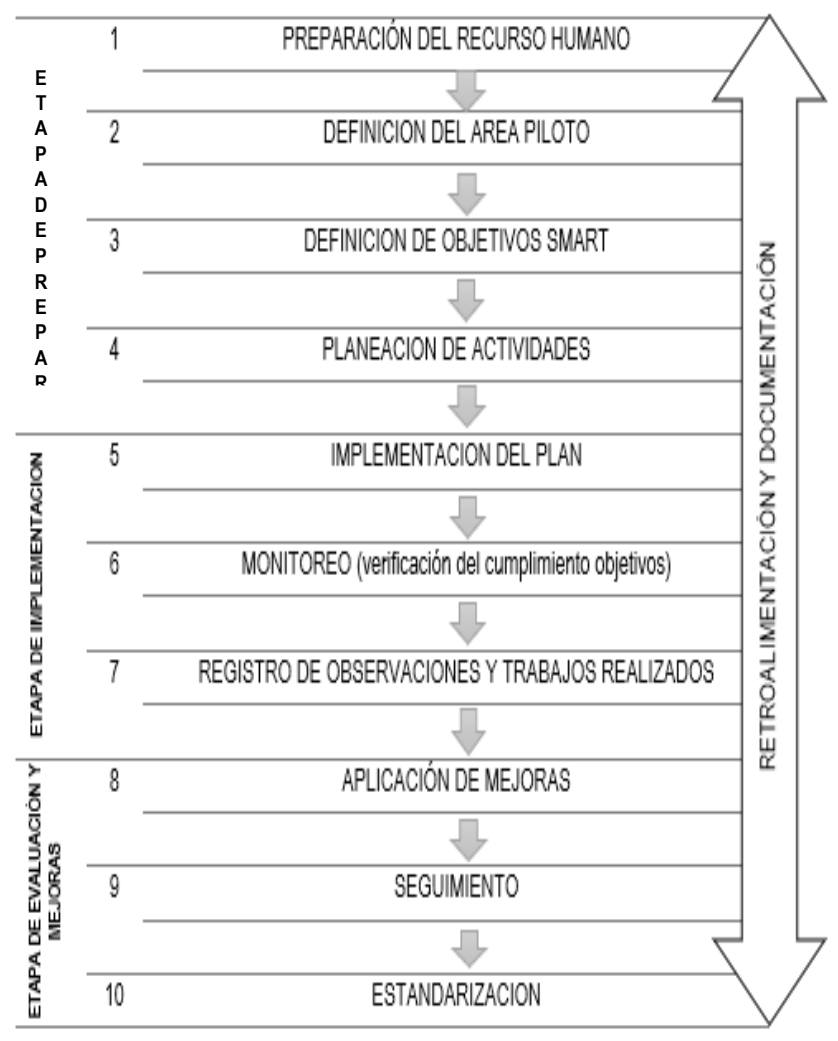

Fig. 8. Metodología basaba en herramientas de RCM, WCM y Lean Manufacturing.

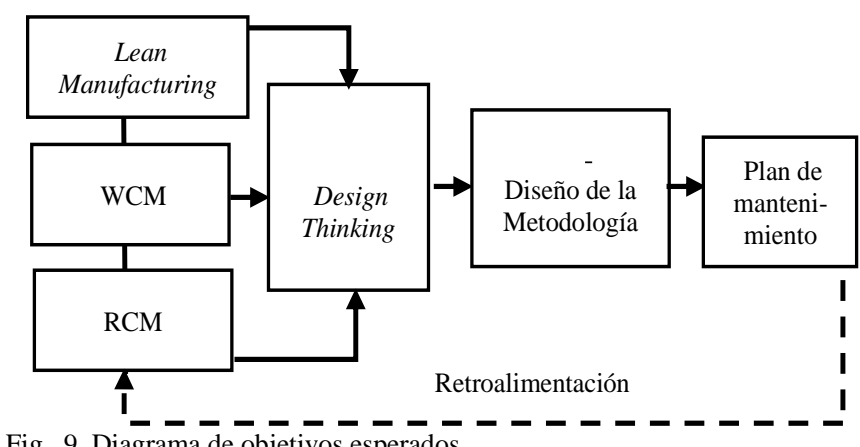

Fig. 9. Diagrama de objetivos esperados. 


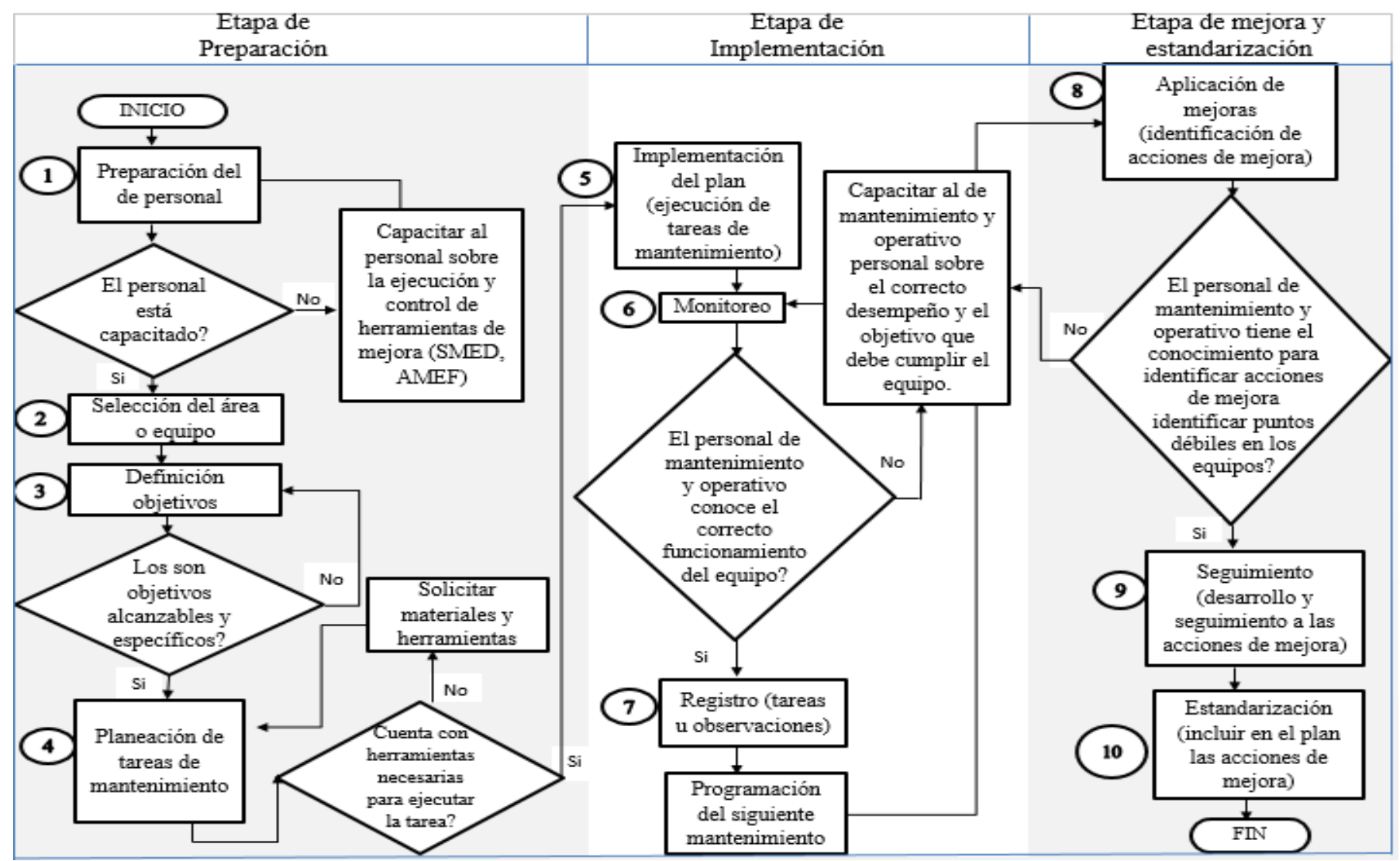

Fig. 10. Diagrama de actividades del plan de mantenimiento [2]

\section{CONCLUSION}

A través de la investigación realizada sobre la aplicación de herramientas de las metodologías RCM, WCM, y Lean Manufacturing en diversos procesos industriales, se logró identificar, seleccionar e integrar un conjunto de dichas para llevar a cabo el diseño de una metodología, la cual se tomó como base para generar un plan de mantenimiento específico para el proceso de trefilado de alambrón de una empresa mexicana del sector del acero como caso de estudio particular. Para llevar a cabo el diseño de dicha metodología fue necesario desarrollar una serie de fases, la cuales fueron basadas en el Design Thinking (empatizar, definir, idear, prototipar, evaluar).

Durante desarrollo de las fases mencionadas fue posible integrar las herramientas de RCM, WCM, y Lean Manufacturing las cuales fueron seleccionadas acorde al objetivo planteado y al problema identificado. Como resultado de esa integración se generó la metodología que muestra una serie de pasos para llevar a cabo el plan de mantenimiento. Posteriormente, con base en dicha metodología se elaboró el plan de mantenimiento el cual es representado en un diagrama de flujo en un lenguaje claro y sencillo específicamente para el proceso de trefilado de alambrón de la empresa mexicana.

Lo anterior proporciona a las partes interesadas la importancia de involucrar al personal operativo en la generación de ideas para mantener el funcionamiento adecuado de los equipos y mejorar los procedimientos para la ejecución de las tareas de mantenimiento haciendo uso de los prototipos (diagramas o esquemas) para lograr del flujo continuo de la producción.

\section{AGRADECIMIENTOS}

Al Consejo Nacional de Ciencia y Tecnología (CONACyT) por contribuir en el financiamiento de estudios de maestría en Ingeniería Industrial y a la Escuela Colombiana de Ingeniería Julio Gravito por fortalecer el desarrollo profesional a través del convenio de doble titulación con el Tecnológico Nacional de México.

\section{REFERENCIAS}

[1] Ortega, M.S. and P.B. Ceballos, Design thinking: Lidera el presente. Crea el futuro. 2015: ESIC Editorial.

[2] Pérez, E., et al., Diseño de una metodología para generar un plan de mantenimiento a través de la integración de Reliability Centered Maintenance, World Class Manufaturing y Lean Manufacturing aplicable en procesos de trefilado de alambrón, 2019.

[3] Arrieta, P.J.G., et al., Benchmarking sobre Manufactura Esbelta (Lean Manufacturing) en el sector de la confección en la ciudad de Medellín, Colombia. Journal of Economics, Finance and Administrative Science, 2010. 15(28): p. 141-170.

[4] Yamashina, H., K. Stefan, and L. Massone, World Class Manufacturing, Methods and Tools for the Fiat Group Automobiles Production System. 2013.

[5] De Felice, F., A. Petrillo, and S. Monfreda, Improving operations performance with world class manufacturing technique: a case in automotive industry, in Operations management. 2013, IntechOpen.

[6] Barajas, A.M. and G.C. Cruz, Propuesta de una estrategia de mantenimiento utilizando Mantenimiento Centrado en Confiabilidad. 2011. 
[7] SAE JA 1011, N., Criterios de Evaluación para Procesos de Mantenimiento centrado en Confiabilidad. Norma para vehículos aeroespaciales y de superficie. ISO estándar: Ginebra, Suiza, 1999.

[8] Gajdzik, B., World Class Manufacturing in metallurgical enterprise. Metalurgija, 2013. 52(1): p. 131-134.

[9] Maldonado Villalva, G., Herramientas y técnicas lean manufacturing en sistemas de producción y calidad. 2008.

[10] Posada, J.G.A., Interacción y conexiones entre las técnicas 5s, SMED y Poka Yoke en procesos de mejoramiento continuo. Tecnura, 2007. 10(20): p. 139-148.

[11] Tapia Coronado, J., et al., Marco de Referencia de la Aplicación de Manufactura Esbelta en la Industria. Ciencia \& trabajo, 2017. 19(60): p. 171-178.

[12] Marchwinski, C. and J. Shook, Léxico lean: glossário ilustrado para praticantes do pensamento lean. 2003: Lean Institute Brasil.

[13] Arrieta, J.G., et al., Aplicación lean manufacturing en la industria colombiana. Revisión de literatura en tesis y proyectos de grado. Revista Virtual Pro, 2011. 132.

[14] Felizzola, J.H. and A.C. Luna, Lean Six Sigma en pequeñas y medianas empresas: un enfoque metodológico. Ingeniare. Revista chilena de ingeniería, 2014. 22(2): p. 263-277.

[15] Mora, L.A., Mantenimiento-planeación, ejecución y control. 2009: Alfaomega Grupo Editor.

[16] Moubray, J., Mantenimiento centrado en confiabilidad. Gran Bretaña: Aladon ltda, 2004.

[17] Fernández, F.J.G., Teoría y práctica del mantenimiento industrial avanzado. 2005: FC editorial.

[18] Posada, J.G.A., V.E.B. Herrera, and M.J.R. Martínez, Benchmarking sobre Manufactura Esbelta (Lean Manufacturing) en el sector de la confección en la ciudad de Medellín, Colombia. The bi-annual academic publication of Universidad ESAN, 2010. 15(28).

[19] Borges, R.C., S.C.A. De Abreu, and J.M. Vaz, Estudo do SMED por meio da metodologia World Class Manufacturing Seminários em Administração, 2014.

[20] Deshpande, V. and J. Modak, Application of RCM to a medium scale industry. Reliability Engineering \& System Safety, 2002. 77(1): p. 3143.

[21] Dacheng, 1. and G. Jinji, Study and application of Reliability-centered Maintenance considering Radical Maintenance. Journal of Loss Prevention in the Process Industries, 2010. 23(5): p. 622-629.

[22] Gang, N., B.-S. Yang, and M. Pecht, Development of an optimized condition-based maintenance system by data fusion and reliabilitycentered maintenance. Reliability Engineering \& System Safety, 2010. 95(7): p. 786-796.

[23] Carazas, F., C. Salazar, and G.F.M.d. Souza, Availability analysis of heat recovery steam generators used in thermal power plants. Energy, 2011. 36(6): p. 3855-3870.

[24] Gonzalez, F., Miniguía: una introducción al Design Thinking+ Bootcamp bootleg. Hasso Platner, Institute of design at Stanford, 2012.

[25] Tejeda, A.S., Mejoras de Lean Manufacturing en los sistemas productivos. Ciencia y sociedad, 2011.

[26] Carazas, F., C. Salazar, and G. Souza, Availability analysis of heat recovery steam generators used in thermal power plants. Energy, 2011. 36(6): p. 3855-3870.

[27] Rubrich, L. and M. Watson, Implementing World Class Manufacturing: A Bridge to Your Manufacturing Survival: Shop Floor Manual. 1998: Wcm Associates.

[28] Li, D. and J. Gao, Study and application of Reliability-centered Maintenance considering Radical Maintenance. Journal of Loss Prevention in the Process Industries, 2010. 23(5): p. 622-629.

[29] Niu, G., B.-S. Yang, and M. Pecht, Development of an optimized condition-based maintenance system by data fusion and reliabilitycentered maintenance. Reliability Engineering \& System Safety, 2010. 95(7): p. 786-796.

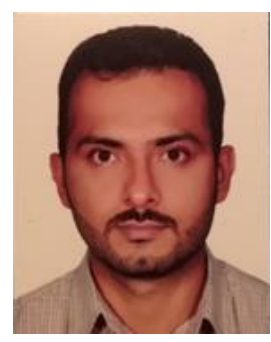

Emilio Pérez Adán. Es Ingeniero Industrial del Instituto Tecnológico de Nuevo León, México. Titulado en el Instituto Tecnológico Superior de Tantoyuca, México (2005-2011). Maestro en Ingeniería Industrial del Instituto Tecnológico Superior de Tantoyuca, Veracruz, México (20172019), Magíster en Ingeniería Industrial de la Escuela Colombiana de Ingeniería Julio Garavito, Bogotá D.C. Colombia (2018-2019). Experiencia laboral en la maquiladora NOVALINK en 2004 en Matamoros, México, Bebidas Mundiales COCA-COLA en 2005 en Guadalupe, Nuevo León. México, Porcelanite LAMOSA S.A.de C.V. en 2008 Villa Juárez, Nuevo León, México. Vigas Pretensadas VELOSA en 2011 en Escobedo, Nuevo León, México. Se desempeñó como docente en el Instituto Tecnológico Superior de Tantoyuca, México en 2012 en el área de Ingeniería en Gestión Empresarial e Ingeniería Industrial.Actualmente profesor en el área de Ingeniería Industrial en la universidad del Atlántico, Tampico, Tamaulipas, México. Representante de ventas para empresa Ilumiled, Puebla, México.

ORCID: https://orcid.org/0000-0002-8000-257X

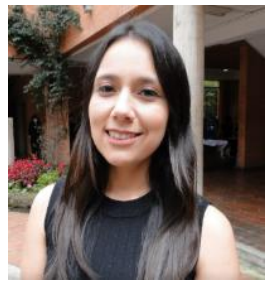

Ivonne Angélica Castiblanco-Jiménez. Ing. Electrónica en 2011 de la Pontificia Universidad Javeriana, Bogotá, Colombia, MSc. en Ing. Mecatrónica en 2011 del Politécnico di Torino, Turín, Italia, MSc. en Automatización Industrial en 2014 del Politécnico di Torino, Turín, Italia. Esp. en Lean Manufacturing y World Class Manufacturing. Experiencia en el campo de gestión de instalaciones, desarrollo de producto y producción para el grupo FIAT CHRYSLER en Turín, Italia. Actualmente investigadora del Politécnico di 25Torino, Turin, Italia, en el centro de investigación 3DLab. Candidata a PhD.

ORCID: 0000-0001 -5866-078X

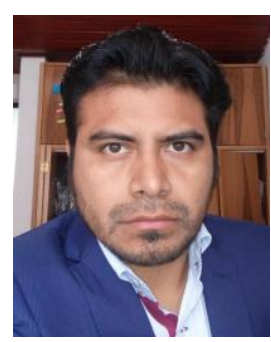

Nicolás Francisco Mateo Díaz. Ingeniero Industrial con especialidad en Sistemas de Manufactura y Productividad (2004-2009); maestría en Ingeniería Industrial en la Línea de Generación y Aplicación de Conocimiento "Diseño y Mejora de Productos y Procesos" (2011-2013), ambos títulos por el Instituto Tecnológico Superior de Tantoyuca, Veracruz, México. Ha sido profesor de Licenciatura en Ingeniería Industrial y del Programa de Maestría en Ingeniería Industrial reconocido por CONACYT. Ha recibido el reconocimiento de Perfil Deseable del PRODEP y también ha sido líder del cuerpo académico en formación "Metodologías Integradas para la Innovación y Desarrollo Tecnológico enfocado a la Industria 4.0". Se ha desempeñado como Subdirector de Posgrado e Investigación, así como Subdirector de Estudios Superiores en el Instituto Tecnológico Superior de Tantoyuca perteneciente al sistema Tecnológico Nacional de México (TecNM). Actualmente es Profesor Investigador de Tiempo Completo del Departamento de Ciencias Básicas e Ingeniería- Ingeniería Industrial- en la Universidad del Caribe, Cancún, Quintana Roo, México.

ORCID: https://orcid.org/0000-0003-4799-6434. 